\title{
High Incidence of Arterial Dissection Associated With Left Vertebral Artery of Aortic Origin
}

\author{
Masaki KOMIYAMA, Toshie MORIKAWA, Hideki NAKAJIMA, \\ Misao NISHIKAWA, and Toshihiro YASUI
}

Department of Neurosurgery, Osaka City General Hospital, Osaka

\begin{abstract}
The incidences of arterial dissection of the vertebral artery (VA) of aortic origin and VA of subclavian artery origin were determined. The origins of the left and right VAs were confirmed by angiography in 860 and 717 patients, respectively. Left VA of aortic origin was found in 21 patients (6 females and 15 males) but no right VA of aortic origin was found. Left VA of left subclavian artery origin was found in 837 patients and right VA of right subclavian artery origin in 717 patients. Arterial dissection of the VA occurred in 17 patients (6 females and 11 males), four patients with left VA of aortic origin, seven with left VA of left subclavian artery origin, four with right VA of right subclavian artery origin, and two with bilateral VAs of subclavian artery origin. Left VA of aortic origin (4 of 21 patients) was associated with a significantly higher incidence of VA dissection than left VA of left subclavian artery origin and right VA of right subclavian artery origin $(p<0.001)$. Left VA of aortic origin is associated with a predilection for VA dissection in comparison to VA of subclavian artery origin.
\end{abstract}

Key words: angiography, arterial dissection, vertebral artery

\section{Introduction}

Spontaneous arterial dissection of the vertebral artery (VA) has been increasingly recognized in recent years. ${ }^{3,7,15,18)}$ Extracranial VA dissection causes brain ischemia, and intracranial dissection causes either subarachnoid hemorrhage or brain ischemia. Vascular anomalies of the VA, including fenestration, duplication, abnormal course, or abnormal origin, are occasionally associated with intracranial vascular lesions, such as cerebral aneurysms, arteriovenous malformation, arterial dissection, and moyamoya disease. ${ }^{5,8,10,12,13,17)}$ We examined the incidence of arterial dissection of the VA and its relationship to the origin of the VA from either the aorta or subclavian artery.

\section{Materials and Methods}

This study retrospectively reviewed 1567 cerebral catheter angiography procedures performed in 1109 patients for many reasons over the past 5 years. Aortography, subclavian or innominate arteriography, or vertebral arteriography was performed in 908 patients. The origins of the left and right VAs were

Received May 1, 2000; Accepted August 28, 2000 confirmed in 860 and 717 patients, respectively. The angiograms of these patients were examined to evaluate the relationship between arterial dissection of the intracranial and/or extracranial VAs and the origin of the VA from the aorta or subclavian artery. The angiographic definition of arterial dissection includes stenosis (string sign), fusiform dilatation, pearl and string sign, occlusion, irregularity of the arterial wall, double lumen sign, stagnation of the contrast material, and chronological change of these signs and/or findings.

\section{Results}

Left VA of aortic origin occurred in 21 patients (6 females and 15 males), an incidence of $2.4 \%$. No right VA of aortic origin was found. Left VA of left subclavian artery origin was found in 837 patients and right VA of right subclavian artery origin in 717 patients. Two patients had dual origin of the left VA, with one leg originating from the aorta and another leg from the left subclavian artery. ${ }^{11)}$ One patient had dual origin of the right VA, with both legs originating from the right subclavian artery.

Arterial dissection of the VA was detected in 17 patients (6 females and 11 males), an incidence of $1.9 \%$. Arterial dissection occurred on the left side in 

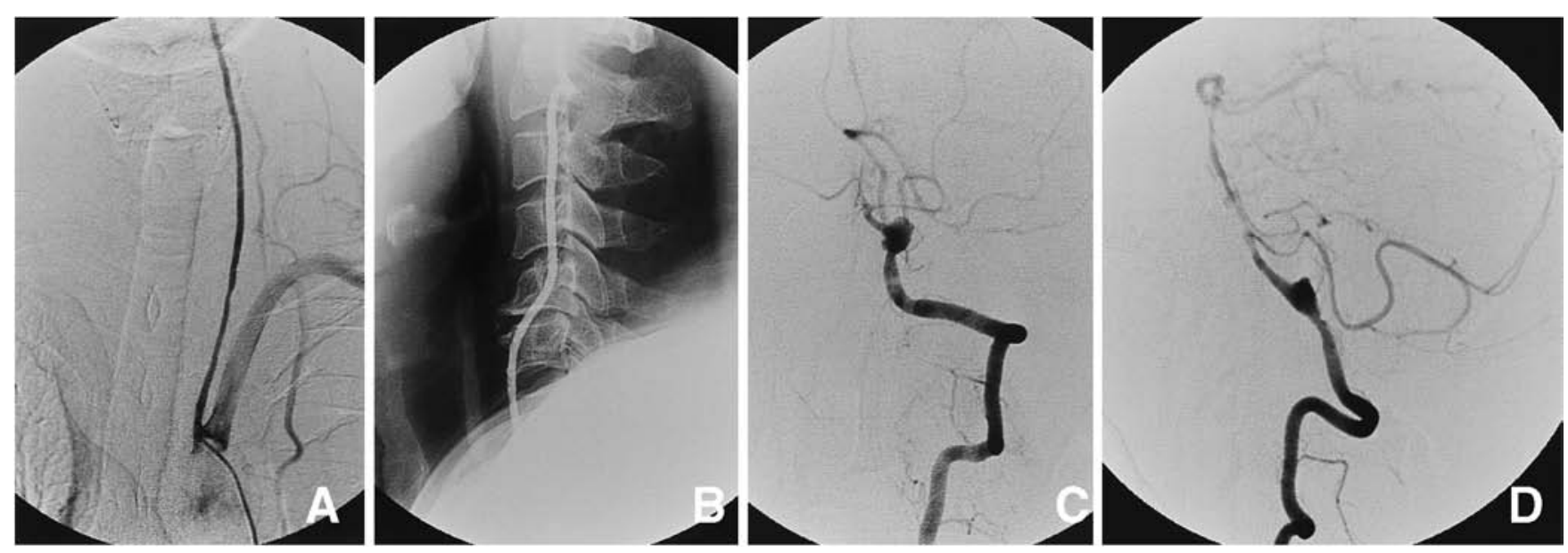

Fig. 1 Case 1. Selective angiograms of the left vertebral artery of aortic origin (A: frontal view, B: lateral view) showing the left vertebral artery entering the transverse foramen at C-5. Intracranial left vertebral angiograms (C: frontal view, D: lateral view) showing string and pearl sign, indicating vertebral dissection.

11 , on the right side in four, and on both sides in two patients. Intracranial dissection was present in 13 patients. Two patients had extracranial dissection and another two patients had both intracranial and extracranial dissection. Nine patients had subarachnoid hemorrhage, seven patients had brain ischemia, and one was asymptomatic.

Four of the 21 patients (19.0\%) with left VA of aortic origin (excluding two patients with dual origin of the VA) developed arterial dissection of the left VA. Nine of the 837 patients with left VA of left subclavian artery origin (1.1\%) developed arterial dissection of the left VA. Six of the 717 patients $(0.84 \%)$ with the right VA of right subclavian artery origin developed arterial dissection of the right VA. Left VA of aortic origin was associated with a significantly higher incidence of arterial dissection of its own vessel than left VA of left subclavian artery origin ( $p<0.001$ ) and right VA of right subclavian artery origin $(\mathrm{p}<0.001)$.

\section{Illustrated Cases}

Case 1: A 53-year-old male developed sudden occipitalgia without neurological deficits. Low-grade fever continued until the patient came to our hospital 10 days later. On admission, the patient was alert without focal deficits except for stiff neck. Computed tomography (CT) showed no abnormalities, but cerebrospinal fluid (CSF) examination revealed xanthochromic CSF. Cerebral angiography revealed arterial dissection of the left VA, which originated directly from the aorta and entered the fifth transverse foramen (Fig. 1). The right VA originated from the right subclavian artery and entered the sixth transverse foramen. The left VA dissection was trapped surgically without sequelae.

Case 2: A 62-year-old male suddenly developed left occipitalgia and then consciousness disturbance. On admission, the patient was drowsy, but responded correctly. He had left Horner's sign, mild left cerebellar sign, and left facial and right hemicorporeal hypalgesia without motor weakness, which was consistent with a diagnosis of left lateral medullary syndrome. CT showed no abnormalities. Cerebral angiography revealed stenotic change (string sign) of the intracranial left VA, from which the left posterior inferior cerebellar artery (PICA) originated. The left VA originated directly from the aorta and entered the fifth transverse foramen, and the right VA originated from the right subclavian artery and entered the sixth transverse foramen (Fig. 2). This patient was treated conservatively. Follow-up angiography on day 15 revealed occlusion of the left VA distal to the left PICA. The patient's consciousness soon recovered completely, but moderate cerebellar sign and sensory deficits remained.

\section{Discussion}

Arterial dissection of the VA occurred more frequently on the left than on the right in our series (11 on the left, 4 on the right, and two bilaterally). Previous reports have not found any apparent predilection in the laterality of intracranial VA dissection: 14 left, 10 right $^{18)}$; 14 left, 17 right, two bilateral ${ }^{15)}$; 124 



Fig. 2 Case 2. Subselective angiograms of the left vertebral artery of aortic origin (A: frontal view, B: lateral view) showing the left vertebral artery entering the transverse foramen at C-5. Intracranial left vertebral angiograms (C: frontal view, D: lateral view) showing the stenotic lesion (arrow) at the origin of the posterior inferior cerebellar artery.

left, 137 right $^{19)}$; or in extracranial VA dissection: six left, four right, five bilateral. ${ }^{3)}$ However, intracranial VA dissection causing hemorrhage occurred more frequently on the right than on the left (left 62, right 98), and dissection causing ischemia occurred more frequently on the left than on the right (left 62, right 39) when examined separately. ${ }^{19)}$

The reasons for the high incidence of arterial dissection associated with VA of aortic origin remain to be elucidated, although statistical chance is possible due to small sample size of the patients with arterial dissection of the VA of aortic origin. However, there could be two anatomical explanations: congenital structural defects of arterial wall and alteration of cerebral hemodynamics. Congenital medial defects of arterial wall occur at the fenestration of the basilar artery. ${ }^{2,4)}$ Such medial defect as well as hemodynamic stress and turbulent flow at the fenestration may contribute to the development of saccular aneurysm at this location. ${ }^{6}$ Embryonic variations of the cerebral arteries, such as duplication of the middle cerebral artery and accessory middle cerebral artery, are often associated with cerebral aneurysms, suggesting a congenital medial defect of the cerebral arteries. ${ }^{9)}$ Similarly, arterial dissection of the VA of aortic origin could be attributable to the structural defects of the VA. Structural defect of the VA, although not proved in the cases of VA of aortic origin, could cause arterial dissection of the VA, usually in the intracranial $\mathrm{V}_{4}$ portions.

The VA is formed in the embryo from the longitudinal vascular anastomoses between the proat- lantal segmental arteries and the seven cervical segmental arteries, which originate from the paired dorsal aortas and supply the spinal cord and eight cervical nerves. ${ }^{14,16)}$ The first six segmental arteries (the first being the proatlantal segmental arteries) involute and the seventh segmental arteries, i.e., the sixth cervical segmental arteries, usually become the proximal portions of the VAs and subclavian arteries. ${ }^{14)}$ Normally, the left VA originates from the left subclavian artery and enters the sixth transverse foramen. However, the left VA occasionally originates directly from the aorta with an incidence of $2.4-5.8 \% .^{7)}$ In this situation, the VA commonly enters the fifth transverse foramen.

Anomalous origin of the VA causes alteration of the cerebral hemodynamics. ${ }^{1)}$ Shear stress may be larger in the VA of aortic origin than in the VA of subclavian artery origin, possibly due to the anatomical differences. The VA of aortic origin may receive direct arterial pulsatile flow whereas the VA of subclavian artery origin may receive damped blood flow due to the presence of the proximal subclavian artery. Different levels of entry of the VA to the transverse foramen may also contribute to differences in hemodynamics. The VA of aortic origin usually enters at the C5-6 intervertebral level whereas the VA of subclavian artery origin enters at the C6-7 intervertebral level. This higher entry of the VA to the transverse foramen may cause larger shear stress in the distal portion of the VA.

Left VA of aortic origin is associated with a predilection for VA dissection in comparison to left 
VA of subclavian artery origin.

\section{References}

1) Bernardi L, Dettori P: Angiographic study of a rare anomalous origin of the vertebral artery. Neuroradiology 9: 43-47, 1975

2) Black SPW, Ansbacher LE: Saccular aneurysm associated with segmental duplication of the basilar artery. J Neurosurg 61: 1005-1008, 1984

3) Chiras J, Marciano S, Vega Molina J, Touboul J, Poirier B, Bories J: Spontaneous dissecting aneurysm of the extracranial vertebral artery (20 cases). Neuroradiology 27: 327-333, 1985

4) Crompton MR: The pathology of ruptured middle cerebral aneurysms with special reference to the differences between the sexes. Lancet 2: 421-425, 1962

5) Eisenberg RA, Vines FS, Taylor SB: Bifid origin of the left vertebral artery. Radiology 159: 429-430, 1986

6) Ferguson GG: Physical factors in the initiation, growth, and rupture of human intracranial saccular aneurysms. J Neurosurg 37: 666-677, 1972

7) Haughton VM, Rosenbaum AE: The normal and anomalous aortic arch and brachiocephalic arteries, in Newton TH, Potts DG (eds): Radiology of the Skull and Brain, vol 2. St Louis, Mosby, 1974, pp 1145-1163

8) Komiyama M, Morikawa T, Nakajima H, Nishikawa M, Yasui T: Embolic cerebellar infarction caused by spontaneous dissection of the extracranial vertebral artery. Two case reports. Neurol Med Chir (Tokyo) 40: 310-314, 2000

9) Komiyama M, Nakajima H, Nishikawa M, Yasui T: Middle cerebral artery variations: duplicated and accessory arteries. AJNR Am J Neuroradiol 19: 45-49, 1996

10) Komiyama M, Nakajima $H$, Nishikawa $M$, Yasui $T$, Kan M: Treatment of a saccular aneurysm at the fenestration of the intracranial vertebral artery with Guglielmi detachable coils. Acta Neurochir (Wien) 141: 1125-1127, 1999

11) Komiyama $M$, Nakajima $H$, Yamanaka $K$, Iwai $Y$ : Dual origin of the vertebral artery. Case report. Neurol Med Chir (Tokyo) 39: 932-937, 1999

12) Kowada M, Yamaguchi K, Takahashi H: Fenestration of the vertebral artery with a review of 23 cases in Japan. Radiology 103: 343-346, 1972

13) Kuwahara T, Aoto K, Uno J, Kagawa T, Sekimoto $H$, Kagawa R: [Duplicate origin of the vertebral artery associated with moyamoya disease]. Rinsho To Kenkyu 66: 1174-1178, 1989 (Jpn)

14) Newton TH, Mani RL: The vertebral artery, in Newton TH, Potts DG (eds): Radiology of the Skull and Brain, vol 2. St Louis, Mosby, 1974, pp 1659-1709

15) Onda H, Tanikawa T, Takeshita M, Arai K, Kawamata T, Ujiie H, Izawa M, Kagawa M, Takakura $\mathrm{K}$ : [Management for dissecting aneurysms of the vertebral artery]. Nosocchu No Geka 22: 293-299, 1994 (Jpn, with Eng abstract)
16) Padget DH: Designation of the embryonic intersegmental arteries in reference to the vertebral artery and subclavian stem. Anat Rec 119: 349-356, 1954

17) Sugita S, Abe T, Okura A, Watanabe M, Shigemori M: MRI demonstration of double origin of the left vertebral artery: case note. Neuroradiology 37: 295-296, 1995

18) Yamaura A, Watanabe $Y$, Saeki N: Dissecting aneurysms of the intracranial vertebral artery. J Neurosurg 72: 183-188, 1990

19) Yamaura A, Yoshimoto $T$, Hashimoto N, Ono J: [Nationwide study of nontraumatic intracranial arterial dissection: clinical features and outcome]. Nosocchu No Geka 26: 79-86, 1998 (Jpn, with Eng abstract)

Address reprint requests to: M. Komiyama, M.D., Department of Neurosurgery, Osaka City General Hospital, 2-13-22 Miyakojima-Hondohri, Miyakojima-ku, Osaka 534-0021, Japan.

\section{Commentary}

The authors have provided a concise and well written article documenting some interesting observations on vertebral artery dissection from their analysis of 860 angiograms on 717 patients. In many instances, the etiology of vertebral artery dissection remains enigmatic. The authors have demonstrated that an aortic origin of the left vertebral artery, although uncommon, is associated with a significantly higher incidence of vertebral artery dissection than a subclavian origin of the vertebral artery. Although this observation could be entirely coincidental, the markedly increased incidence of vertebral artery dissection with aortic as opposed to subclavian origin in this study provides compelling evidence that there may be a hemodynamic or embryologic relationship between an aortic origin of the vertebral artery and predisposition to dissection. This outstanding study should stimulate further investigation of this potential association.

Daniel L. BARROW, M.D. Department of Neurosurgery Emory University School of Medicine The Emory Clinic Atlanta, Georgia, U.S.A.

This study examined the incidence of a direct origin of the left vertebral artery (VA) from the aorta, and the relative incidence of VA dissection, in a large number of patients with vertebral angiograms accumulated over five years. The interesting finding made was that there was a 19\% incidence of VA dissection in the 21 
patients with left VA aortic origin, compared with around $1 \%$ where the left or right VA arose from the subclavian. Although the numbers were small, the difference was highly significant and considerable, with an odds ratio of 21.7 (95\% confidence interval 6.1-77.3). Possible explanations for this difference are well discussed, including possible structural anomalies in the wall of the vessel, known to be associated with other congenital vascular anomalies, and greater haemodynamic stress in the artery arising directly from the aorta with its higher pressure and pulsation. Extensions of this work from other large series would be interesting, including whether there is any association with aneurysm formation or other vessel abnormalities.

Nicholas W. C. Dorsch, M.D., F.R.C.S., F.R.A.C.S. Department of Surgery Westmead Hospital Sydney, Australia

Komiyama et al. attempted to correlate the incidence of arterial dissection of the vertebral artery (VA) and its relationship to the origin of the VA in 1,577 patients with vertebral angiography. This interesting study suggested that left VA of aortic origin was associated with a predilection for VA dissection (4 of 21 patients) in comparison to left VA of left subclavian artery origin (7 of 837 patients), and right VA of right subclavian artery origin ( 4 of 717 patients). Although statistical chance is possible due to the small sample size of the patients with arterial dissection of the VA of aortic origin (4 patients), the authors tried to interpret the finding as a result of altered cerebral hemodynamics and shear stress caused by an anomalous origin of the VA. As the patients with dissecting aneurysms were characteristically relatively young males, and a right-sided dominance was reported, ${ }^{1,2)}$ further accumulation of such cases would be necessary to elucidate this unique VA lesion.

\section{References}

1) Berger MS, Wilson CB: Intracranial dissecting aneurysms of the posterior circulation. Report of six cases and review of the literature. J Neurosurg 61: 882-894, 1984

2) Manz HJ, Lussenhop AJ: Dissecting aneurysm of intracranial vertebral artery: case report and review of literature. J Neurol 230: 25-35, 1983

Kyu Chang LEE, M.D. Department of Neurosurgery Yonsei University College of Medicine Seoul, Korea, R.O.K. 